\title{
Author Correction: MYC-driven epigenetic reprogramming favors the onset of tumorigenesis by inducing a stem cell-like state
}

\author{
Vittoria Poli ${ }^{1,2}$, Luca Fagnocchi ${ }^{1,2,3}$, Alessandra Fasciani ${ }^{1,2}$, Alessandro Cherubini ${ }^{2}$, Stefania Mazzoleni (i) ${ }^{2}$, \\ Sara Ferrillo2, Annarita Miluzio ${ }^{2}$, Gabriella Gaudioso $2,3,4$, Valentina Vaira $2,3,4$, Alice Turdo ${ }^{5}$, Miriam Gaggianesi ${ }^{5}$, \\ Aurora Chinnici ${ }^{5}$, Elisa Lipari ${ }^{5}$, Silvio Bicciato ${ }^{6}$, Silvano Bosari ${ }^{3,4}$, Matilde Todaro (iD ${ }^{7}$ \& Alessio Zippo ${ }^{1,2,3}$
}

Correction to: Nature Communications; https://doi.org/10.1038/s41467-018-03264-2; published online 9 March 2018

The original version of this Article contained an error in the spelling of the author Miriam Gaggianesi, which was incorrectly given as Miriam Giaggianesi. Furthermore, the affiliation details for Gabriella Gaudioso, Valentina Vaira and Silvano Bosari incorrectly omitted 'Division of Pathology, Fondazione IRCCS Ca' Granda Ospedale Maggiore Policlinico, Milan, 20122, Italy'. Finally, the affiliation details for Alice Turdo, Miriam Gaggianesi, Aurora Chinnici and Elisa Lipari were incorrectly given as 'Dipartimento di Biotecnologie Mediche e Medicina Legale Sezione di Biochimica Medica, Facoltà di Medicina e Chirurgia, Policlinico "P.Giaccone”, Università di Palermo, Palermo, 90127, Italy'. The correct affiliation is 'Department of Surgical, Oncological and Stomatological Sciences, University of Palermo, Palermo, 90127, Italy'. These errors have now been corrected in both the PDF and HTML versions of the Article.

Published online: 20 September 2018

Open Access This article is licensed under a Creative Commons Attribution 4.0 International License, which permits use, sharing, adaptation, distribution and reproduction in any medium or format, as long as you give appropriate credit to the original author(s) and the source, provide a link to the Creative Commons license, and indicate if changes were made. The images or other third party material in this article are included in the article's Creative Commons license, unless indicated otherwise in a credit line to the material. If material is not included in the article's Creative Commons license and your intended use is not permitted by statutory regulation or exceeds the permitted use, you will need to obtain permission directly from the copyright holder. To view a copy of this license, visit http://creativecommons.org/licenses/by/4.0/.
}

(C) The Author(s) 2018

\footnotetext{
${ }^{1}$ Laboratory of Chromatin Biology \& Epigenetics, Center for Integrative Biology (CIBIO), University of Trento, 38123 Trento, Italy. ${ }^{2}$ Fondazione Istituto Nazionale di Genetica Molecolare "Romeo ed Enrica Invernizzi", Via F. Sforza 35, 20122 Milan, Italy. ${ }^{3}$ Division of Pathology, Fondazione IRCCS Ca' Granda Ospedale Maggiore Policlinico, Milan 20122, Italy. ${ }^{4}$ Department of Pathophysiology and Transplantation, University of Milan, Milan 20122 , Italy.

${ }^{5}$ Department of Surgical, Oncological and Stomatological Sciences, University of Palermo, Palermo 90127, Italy. ${ }^{6}$ Center for Genome Research, University of Modena and Reggio Emilia, Modena 41125, Italy. ${ }^{7}$ DiBiMIS, University of Palermo, Palermo 90127, Italy. Correspondence and requests for materials should be addressed to A.Z. (email: alessio.zippo@unitn.it)
} 